\title{
No Risk No Fun: Project-Based Learning in a Nutshell
}

\author{
Ulrike Jaeger
}

Inhalt und Anliegen — Lehre ist im Alltag oft die Performance der Lehrenden. Je besser diese Performance des Alleinverantwortlichen wird, umso besser werden die Zuhörer "versorgt" und umso passiver werden die Lernenden.

Sie verpassen damit aber auch die Erfahrung von Problematik und Komplexität, die wohlmeinende Lehre allzu schnell erklärt. Es fehlt das Erleben, es fehlt die Chance, Fragen zu entwickeln, aus denen erst Interesse und Verständnis entstehen. John Cowan sagt: „Fragen entzünden unser Verständnis, nicht Antworten". Dieses kostbare Fragen, Stutzen, Staunen, wird auch im Theater ausgenutzt, um die Aufmerksamkeit zu lenken, eine Story bedeutsam zu machen. Nachdenken Reflexion, Feedback und neues Ansetzen sind Grundprinzipien einer Lernerfahrung, die nachhaltiger ist als das Wiedergeben passiv aufgenommener Information.

Damit werden die Studierenden zu den eigentlich Handelnden und wir Lehrende haben die Aufgabe, ihnen eine Bühne aufzubauen, auf der sie in gezielt inszenierten Projekten Erfahrungen nicht ausweichen können. Lehrende sind Regisseure für die agierenden Studierenden, die in selbstständig gewählten Teams über möglichst Vieles entscheiden und danach auch handeln, so dass sie sich für ihren Prozess und das Ergebnis verantwortlich fühlen. Diese Eigenverantwortung motiviert intrinsisch.

Das Risiko bei solchen Lernszenarien wird von beiden Seiten gesehen: die Lehrenden fürchten, dass die Projekte nicht gut gestaltet sind oder die Studierenden ihren Freiraum nicht gut nutzen. Studierende fürchten, sich falsch zu entscheiden. Beiden hilft eine gute Organisation und ein ständiger Feedback-Prozess, der Unklarheiten schnell beseitigt. Das reine Fachwissen ist dagegen auch auf andere Weise als durch Unterricht zu vermitteln, und wird von den Studierenden im Austausch und in Recherche sehr selbstständig entdeckt. Und das wollen wir: selbstständiges Lernen als Kompetenz für das gesamte Leben.

Reflexion über den Beitrag zum Scenario-Symposium — In 10 Minuten reinem Vortrag wurde vor allem das Unbehagen an der herkömmlichen ständigen Selbstoptierung der Lehrenden angesprochen und Mut gemacht, es auch einmal anders herum zu versuchen. 
Für den Workshop war es interessant, Lernszenarien in dem technischen Fach der Informatik zu sehen. PBL als Methode ist Jahrzehnte alt, wird aber in Deutschland eher selten angewendet.

Das Symposium selbst brachte darüber hinaus Anregungen performativer Ansätze, die in unserer technischen Disziplin nicht versucht werden. Besonders die körperliche und nonverbale Auseinandersetzung mit Konzepten und Ideen sollten wir auch gegen den Widerstand der Studierenden einführen. Die hervorragende Bedeutung der Reflexion über Gespräche, Lesen/Schreiben von Texten, Zuhören war eine Bestätigung unserer eigenen Erfahrungen in der Lehre. Es lohnt sich und macht Mut, dafür auch hinreichend viel Zeit einzuplanen.

An unserer Hochschule werden bereits in Zusammenarbeit mit den Theaterpädagogen des Theaters Heilbronn, vereinzelt Kurse angeboten, die ...

- ... Theater als Erlebnis anbieten, bei dem u.U. in der Erfahrung klar wird, dass sich das auch auf die berufliche Situation übertragen lässt.

- ... performative Methoden in der Lehre einsetzen, z.B. in Rollenspielen, Gesprächstraining, Vortragstechniken.

Was aber fehlt, ist die Integration dieser Ansätze in das fachliche Curriculum, bei der die Studierenden direkt erleben, wie sinnvoll performatives Arbeiten in ihrem Berufsfeld sein kann. Eine Idee wäre, Tandems aus Fachkollegen und Theaterpädagogen zu bilden, die gemeinsam ein Lernszenario im Kleinen oder für einen ganzen Kurs entwickeln können. 